stances into the middle ear have also been used to induce otitis media in mice (8). Genes that relate to mucin production and other functional aspects of the middle-ear cavity have also been identified $(11,12)$. In addition, the developmental anatomy of the middle ear and its conductive apparatus have been described for many common laboratory species (13-17).

This body of information, along with the identification of the current Eya4 ${ }^{-/-}$mutant mouse as a genetic model of OME (6), provides a rich database for future research on the treatment of chronic OME. This research might identify pharmacological approaches to reduce the long-term effects of effusions in the middle-ear space, or might seek to reduce or reverse cilia damage, polyp formation, and viscosity changes in the accumulated secretions. Treatments that maintain the patency of the ET may also be sought, and all such developments would represent important advances in the treatment of OME.

\section{Acknowledgments}

The National Institute on Deafness and Other Communication Disorders supports E. Lazaridis through training grant DC005363, while J.C. Saunders is supported by research award DC000710. The authors appreciate the comments of Mary Graham, Kirin Kennedy, Matthew Giampapola, and Michael Bilyk.

Address correspondence to: Evelyn Lazaridis, Department of Otorhinolaryngology: Head and Neck Surgery, University of Pennsylvania, 5 Ravdin-ORL, 3400 Spruce Street, Philadelphia, Pennsylvania 19104, USA. Phone: (215) 898-7504; Fax: (215) 898-7504; E-mail: elaz@mail.med.upenn.edu.

1. Rosenfeld, R.M., et al. 2004. Clinical practice guideline: otitis media with effusion. Otolaryngol. Head Neck Surg. 130:S95-S118.

2. Neto, W.C., et al. 2005. Otite média. FORL: Fundacao de Otorrinolaringologia website [In Portuguese]. http://www.forl.org.br/pdf/seminarios/ seminario_34.pdf.

3. Bluestone, C.R., and Klein, J.O. 2003. Otitis media and Eustachian tube dysfunction. In Pediatric otolaryngology. C. Bluestone, et al., editors. Saunders. Philadelphia, Pennsylvania, USA. 474-685.

4. Magnuson, B. 2001. Physiology of the Eustachian tube and middle ear pressure regulation. In Physiology of the ear. A.E. Jahn and J. Santos-Sacchi, editors. Singular. San Diego, California, USA. 75-99.

5. Wayne, S., et al. 2001. Mutations in the transcriptional activator EYA4 cause late-onset deafness at the DFNA10 locus. Hum. Mol. Genet. 195-200.

6. Depreux, F.F.S., et al. 2008. Eya4-deficient mice are a model for heritable otitis media. J. Clin. Invest.
118:651-658.

7. Zheng, Q.Y., Hardisty-Hughes, R., and Brown, S.D 2006. Mouse models as a tool to unravel the genetic basis for human otitis media. Brain Res. 1091:9-15.

8. Ryan, A.F., et al. 2006. Mouse models of induced otitis media [abstract]. Brain Res. 1091:3-8.

9. MacArthur, C.J., and Trune, D.R. 2006. Mouse models of otitis media. Curr. Opin. Otolaryngol. Head Neck Surg. 14:341-346.

10. Vicente, J., et al. 2007. Evolution of middle ear changes after permanent eustachian tube blockage. Arch. Otolaryngol. Head Neck Surg. 133:587-592.

11. Kerschner,J.E. 2007. Mucin gene expression in human middle ear epithelium. Laryngoscope. 117:1666-1676.

12. Elsheikh, M.N., and Malfouz, M.E. 2006. Up-regulation of MUC5AC and MUC5B mucin genes in nasopharygeal respiratory mucosa and selective upregulation of MUC5B in middle ear in pediatric otitis media [abstract]. Laryngoscope. 116:365-369.

13. Vrettakos, P.A., Dear, S.P., and Saunders, J.C. 1988. Middle-ear structure in the chinchilla: a quantitative study. Am. J. Otolaryngol. 9:58-67.

14. Cohen, Y.E., Bacon, C.K., and Saunders, J.C. 1992. Middle-ear development. III: Morphometric changes in the conducting apparatus of the Mongolian gerbil. Hear. Res. 62:187-193.

15. Zimmer, W.M., Rosin, D.F., and Saunders, J.C. 1994 Middle-ear development VI: Maturation of the rat conducting apparatus. Anat. Rec. 239:475-484.

16. Saunders, J.C., and Crumling, M.A. 2001. The structure and function of the outer and middle ear of the mouse. In Handbook of mouse anditory research: from behavior to molecular biology. J. Willott, editor. CRC Press. New York, New York, USA. 99-115.

17. Thomas, J.P., and Walsh, E.J. 1990. Postnatal development of the middle ear: Areal ratios in kittens. Otolaryngol. Head Neck Surg. 103:427-435.

\title{
Revisiting oxidative damage in ALS: microglia, Nox, and mutant SOD1
}

\author{
Séverine Boillée and Don W. Cleveland
}

Ludwig Institute for Cancer Research, Department of Medicine, and Department of Neuroscience, UCSD, La Jolla, California, USA.

\begin{abstract}
Mutation in superoxide dismutase-1 (SOD1) causes the inherited degenerative neurological disease familial amyotrophic lateral sclerosis (ALS), a non-cell-autonomous disease: mutant SOD1 synthesis in motor neurons and microglia drives disease onset and progression, respectively. In this issue of the JCI, Harraz and colleagues demonstrate that SOD1 mutants expressed in human cell lines directly stimulate NADPH oxidase (Nox) by binding to Rac1, resulting in overproduction of damaging ROS (see the related article beginning on page 659). Diminishing ROS by treatment with the microglial Nox inhibitor apocynin or by elimination of Nox extends survival in ALS mice, reviving the proposal that ROS mediate ALS pathogenesis, but with a new twist: it's ROS produced by microglia.
\end{abstract}

Amyotrophic lateral sclerosis (ALS) is the most common adult-onset motor neuron

Nonstandard abbreviations used: ALS, amyotrophic lateral sclerosis; Nox, $\mathrm{NADPH}$ oxidase; $\mathrm{O}_{2}{ }^{-}$, , superoxide; SOD1, superoxide dismutase-1.

Conflict of interest: The authors have declared that no conflict of interest exists.

Citation for this article: J. Clin. Invest. 118:474-478 (2008). doi:10.1172/JCI34613. disease. It is characterized by progressive paralysis and death, usually within 5 years, and no treatment substantially slows disease progression. The majority of ALS cases are sporadic, but among the familial cases of disease, mutations in the superoxide dismutase-1 (SOD1) gene are the most frequently identified. SOD1 catalyzes the conversion of superoxide $\left(\mathrm{O}_{2}{ }^{-}\right)$to $\mathrm{H}_{2} \mathrm{O}_{2}$, and elevation in redox stress has been proposed as a potential cause for motor neuron degeneration. Indeed, SOD1 mimetics have been reported to slow disease progression when administered at disease onset in ALS mice (1). Several hypotheses have been proposed in motor neuron degeneration, including damage within motor neurons and a non-cell autonomous aspect reflecting mutant SOD1-mediated damage within non-neuronal cells (2). Most of the studies supporting these hypotheses have been conducted in transgenic mice and rats in which expression of a mutant SOD1-encoding transgene recapitulates ALS-like disease, including muscle denervation and atrophy, glial activation, and motor neuron death. The importance of glial cell participation in disease was initially shown in chimeric mice in which mutant SOD1-expressing motor neurons 
A

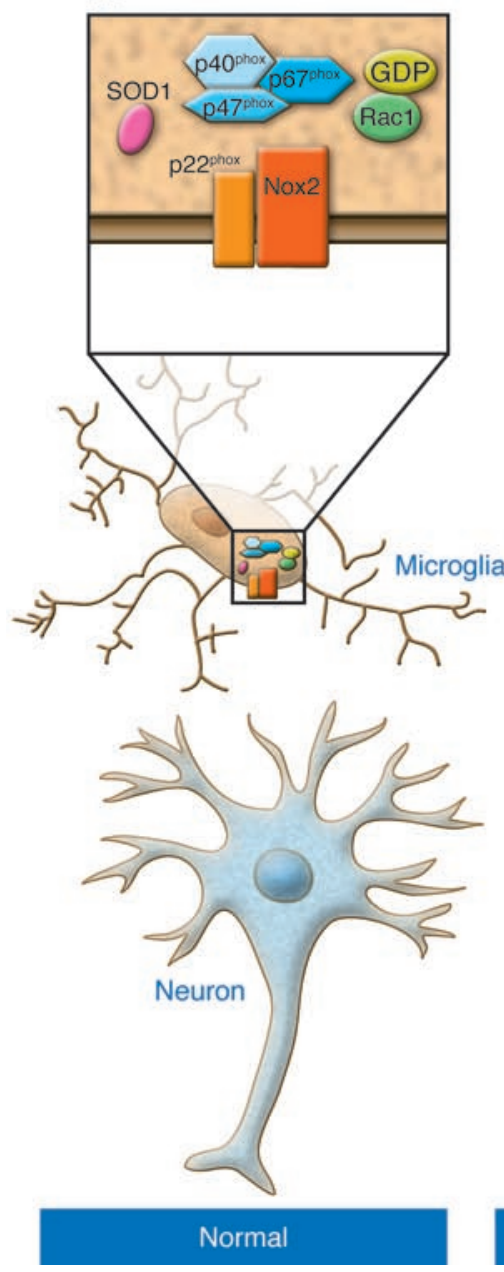

B

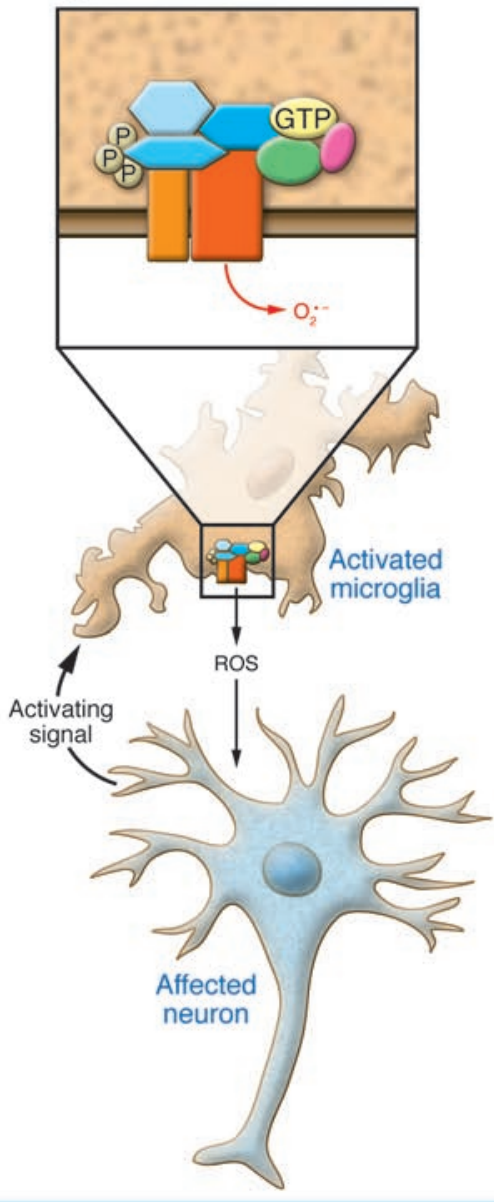

Common neuroinflammatory response
C

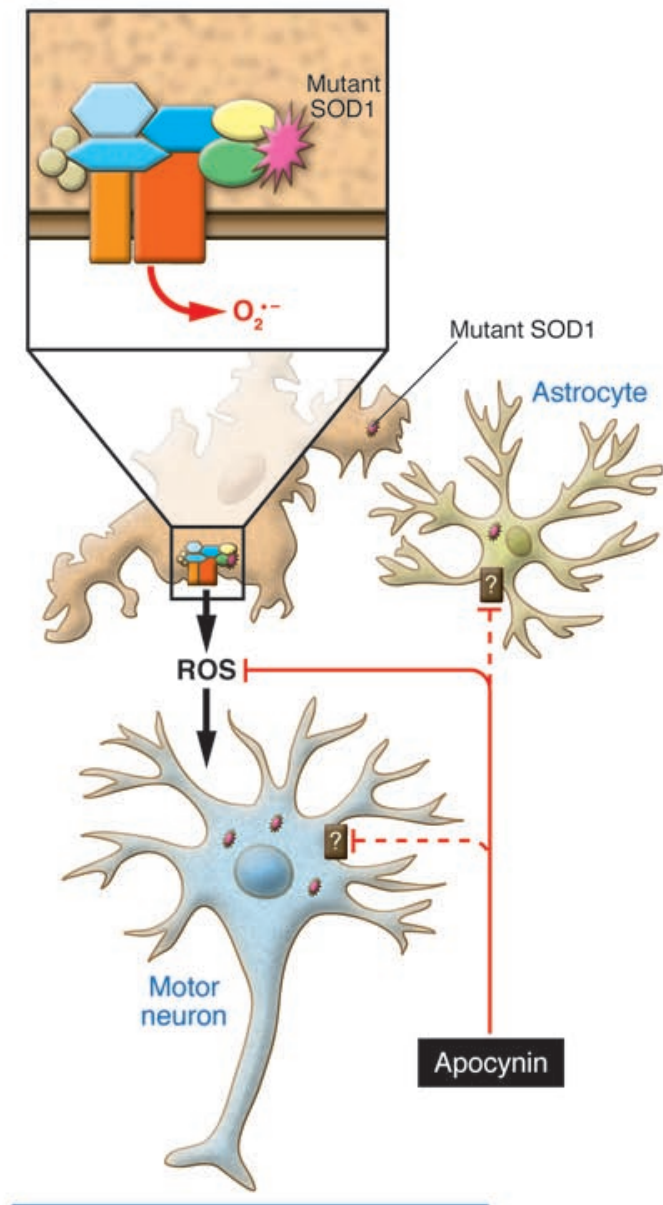

Mutant SOD1-induced ALS

Figure 1

Production of ROS by microglial Nox2 during inflammation is amplified by mutant SOD1 binding to Rac1 in ALS. (A) Under normal conditions, cytosolic activators p67 phox, p47 phox, and p40phox of the microglial Nox catalytic subunit Nox2 are not bound to Nox2, and Rac1 is linked to GDP. (B) Upon microglial activation, including following a stimulus generated from degenerating neurons, Nox is activated and the p47phox subunit becomes phosphorylated and translocates the p67phox/p47phox/p40 phox complex to the membrane. The new report in this issue from Harraz and colleagues (20) shows that SOD1 stabilizes the Nox2 activator Rac1-GTP. The activated Nox complex produces $\mathrm{O}_{2}{ }^{--}$and ROS derived from $\mathrm{O}_{2}{ }^{\cdot-}$, which can in turn damage neurons. (C) In the case of mutant SOD1-induced ALS, Harraz and colleagues show that mutant SOD1 keeps Rac1 in its active GTP-bound form and increases the production of ROS by microglial cells. The drug apocynin blocks microglial production of ROS by inhibiting Nox2 and could potentially block other forms of Nox in additional cell types, including neurons and astrocytes.

were rescued by the presence of normal (non-mutant-expressing) cell neighbors (3). Among these cells in the motor neuron environment, astrocytes have been linked to motor neuron degeneration in ALS because of their reduced capacity for reuptake at synapses of the excitatory neurotransmitter glutamate due to decreased expression of astrocytic glutamate transporters during disease (4). Recently, 3 groups have shown an increased capacity of mutant SOD1-expressing astrocytes to kill motor neurons in culture (5-7). Recognizing the large spectrum of neurotoxic and neurotrophic compounds that they can release, microglia - the inflammatory cells of the CNS - represent the other good candidate cell type for non-cell autonomous killing of neurons. Indeed, minocyclin, an antibiotic also capable of downregulating microglial activation, increased survival in ALS mice $(8,9)$, while the proinflammatory molecule LPS exacerbated disease (10). Even more compelling are data obtained by selective silencing of mutant SOD1 synthesis by microglia (11) or replacement of the mutant SOD1expressing cells of the myeloid lineage (including microglial cells) with normal ones by bone marrow transplantation of their precursors (12). Both approaches demonstrate that mutant SOD1 synthesis in inflammatory cells of the CNS directly accelerates the rate of disease progression. However, our understanding of the means used by these glial cells to kill motor neurons is still in its infancy.

Recently, 2 groups, including that of Harraz, Engelhardt, and colleagues, have followed up on the notion that oxygen radicals produced in microglial cells by NADPH oxidases (Noxs) are a part of microglial toxicity $(13,14)$. By crossing mice expressing the SOD1 ${ }^{\mathrm{G} 93 \mathrm{~A}}$ mutant with mice lacking a catalytic subunit of Nox (through deletion 
of the gene encoding either Nox1 or Nox2), an increase in survival was seen, with Nox2 deletion proving to be of greater benefit than Nox1 deletion (14). Consistent with progressive microglial activation during disease, Nox2 expression was upregulated in SOD $1^{\mathrm{G} 93 \mathrm{~A}}$ mice $(13,14)$ and sporadic ALS patients (13), with the former leading to increased $\mathrm{O}_{2}{ }^{\circ-}$ generation in mouse spinal cord tissues (14).

\section{Nox: a superproducer of $\mathrm{O}_{2}{ }^{--}$}

Nox is a transmembrane protein that transports electrons across biological membranes to reduce oxygen to $\mathrm{O}_{2}{ }^{-}$(15). The Nox family is composed of 7 members; Nox1-Nox5, Duox1, and Duox2 are distributed in a variety of tissues. The Nox prototype, Nox2, is the phagocytic Nox (also known as gp91phox) that generates $\mathrm{O}_{2}{ }^{--}$not as a byproduct, but as a primary function of the enzyme (15). Nox2 contains an NADPH-binding site, a FAD-binding site, and 4 heme-binding histidines. Nox 2 is constitutively associated with the transmembrane Nox stabilizing protein $\mathrm{p} 22^{\text {phox }}$, and recruitment of the activating cytosolic components $\mathrm{p} 47 \mathrm{phox}$, $\mathrm{p} 67 \mathrm{phox}^{\text {, and }} \mathrm{p} 40^{\mathrm{phox}}$ are needed for function (Figure 1). Upon cell activation, p47phox is phosphorylated, thereby initiating translocation of the $\mathrm{p} 47^{\mathrm{phox}} / \mathrm{p} 67_{\mathrm{phox}} / \mathrm{p} 40^{\mathrm{phox}}$ complex to the membrane, where phosphorylated p47phox binds to p22 $2^{\text {phox }}$. Another level of regulation comes from members of the Rho family of small GTPases, including the Rac family. Members of the Rac family are not strictly NADPH subunits, as they regulate other cellular processes, in particular cytoskeletal dynamics. Rac acts to coordinate the translocation of the p47phox/p67phox/ p40phox complex and is active only when bound to GTP, not when bound to GDP. The release of GDP to enable a new round of GTP loading is catalyzed by guanine nucleotide exchange factors. Inactivation of Rac is mediated via GTPase-activating proteins, which stimulate the intrinsic ability of Rac to hydrolyze GTP to GDP. Not all Nox family members require the same interacting partners, but Nox 1 and Nox 2 are both p47phox and Rac dependent (15).

\section{Nox participation in neuronal injury is linked to inflammation}

How does Nox participate in the pathogenesis of ALS? Because mutant SOD1-mediated damage within microglia accelerates disease progression $(11,12)$, the simplest view of a potential role for Nox in ALS would be that activation of Nox consequent to microglial cell activation leads to the production of higher levels of $\mathrm{O}_{2}{ }^{--}$. In turn, this would affect and kill already damaged motor neurons. This scenario is consistent with protection observed by Nox 2 deletion in other models of neurodegeneration in which inflammation has been implicated, including ischemia (16) and dopaminergic neuron killing induced by 1-methyl 4-phenyl 1,2,3,6-tetrahydropyridine (MPTP) in a mouse model of Parkinson disease (17). In addition, dopaminergic neurons cocultured with microglial cells lacking the Nox2 gene are more resistant to rotenone-induced neuronal death (18), and Alzheimer disease-linked amyloid peptides (derived from neurons) activate microglial cells to produce more ROS (19). However, in the case of ALS associated with SOD1 mutations, the new data reported by Harraz, Engelhardt, and colleagues in this issue of the JCI (20) provide a direct link between the disease-causing mutant SOD1 and Nox-mediated ROS production by microglial cells, through SOD1 binding to the Nox activator Rac1, thereby influencing the non-cell autonomous killing of motor neurons.

\section{Mutant SOD1 stimulates Rac1-GTP activation of Nox and production of ROS}

Using an approach involving immunoprecipitation of Rac1 from different mouse tissues, Harraz and colleagues proposed that SOD1 interacts directly with Rac1 but not with the other Nox 2 cytosolic regulators (20). It is puzzling why multiple earlier screens for SOD1-interacting proteins, including yeast 2-hybrid approaches, did not identify Rac1. Nevertheless, as Nox produces $\mathrm{O}_{2}{ }^{-}$that is converted to $\mathrm{H}_{2} \mathrm{O}_{2}$ by SOD1, it was further tested whether the enzymatic activity of SOD 1 was important for this interaction of SOD1 with Rac1. Only the metalated (native) form of SOD1 bound to Rac1, while the demetalated (enzymatically inactive) SOD1 did not. In addition, the binding of SOD1 to Rac1 was redox sensitive, and could be cycled between bound and unbound states depending on the redox state of Rac1. Under reducing conditions, SOD1 efficiently bound Rac1GTP, the form of Rac1 that is recruited to Nox2 upon activation. The binding of SOD1 to Rac1-GTP inhibited the intrinsic and/or GAP-facilitated GTPase activity of Rac1 (Figure 1).

It is now widely accepted that ALS-linked mutations in SOD1 provoke disease due to the acquisition of one or more toxic prop- erties of mutant SOD1 (2). In their current study, Harraz, Engelhardt, and colleagues show that $\mathrm{O}_{2}{ }^{--}$production was enhanced in tissues from mice expressing the catalytically active ALS-linked mutant SOD1 ${ }^{\mathrm{G} 93 \mathrm{~A}}$, but not in tissues from mice expressing comparably high levels of SOD $1^{\mathrm{WT}}$ (Figure 1 and ref. 20). This counterintuitive finding that the enzyme whose primary responsibility is to remove $\mathrm{O}_{2}{ }^{-}$- can in fact serve to amplify $\mathrm{O}_{2}{ }^{\circ-}$ production was also seen in glial and neuronal cell lines expressing mutant SOD $1^{\mathrm{G} 93 \mathrm{~A}}$ and SOD $1^{\mathrm{L} 8 \mathrm{Q}}$, but not SOD1 ${ }^{\mathrm{WT}}$, and was accompanied by increased cell death. In addition, liver tissues from SOD $1^{\mathrm{G} 93 \mathrm{~A}}$ mice - in which no altered pathology is normally observed in ALS - also displayed increased production of $\mathrm{O}_{2}{ }^{--}$, in agreement with a direct effect of mutant SOD1 on Nox activation rather than a consequence of increased inflammation, as observed in the spinal cord following motor neuron degeneration. Such a direct effect of increased ROS as a result of mutant SOD1 within microglial cells obviously could influence the survival of motor neurons, in agreement with previous demonstrations of slowed disease progression after reducing or eliminating mutant SOD1 synthesis within the myeloid lineage $(11,12)$.

A link between increased production of $\mathrm{O}_{2}{ }^{-}$- by mutant SOD1 and elevated Rac1GTP levels was seen not just for SOD1 ${ }^{\mathrm{G} 93 \mathrm{~A}}$, but also for 2 additional ALS-linked SOD1 mutants: SOD $1^{\mathrm{L} 8 \mathrm{Q}}$ and SOD $1^{\mathrm{G} 10 \mathrm{~V}}(20)$. It should be recognized that the choice of these additional 2 mutants, which have not been well characterized for their biochemical properties as dismutases or metal binding, leaves a crucial question unsettled: whether increased stabilization of Rac1GTP and corresponding Nox production of $\mathrm{O}_{2}{ }^{-}$is a general property of the more than 115 different mutations in the SOD1 gene known to cause ALS. A multiplicity of prior evidence established that toxicity is independent of dismutase activity, with multiple mutants (including SOD $1^{\mathrm{G} 85 \mathrm{R}}$, SOD $1^{\mathrm{H} 46 \mathrm{R}}$, and SOD $1^{\mathrm{G} 127 \mathrm{X}}$ ) shown to be causative for disease in humans and rodents but completely inactive as dismutases (2). Further, the fastest rates of disease progression in humans are observed in individuals possessing the least biochemically stable mutants, which accumulate at the lowest levels (21), some of which are almost certainly very poorly folded and inactive as dismutases. Similarly, the failure of demetalated SOD $1^{\mathrm{WT}}$ to bind and activate Rac1-GTP 
would predict that removal of the copper chaperone for SOD1 (CCS) would substantially slow disease, but this was not the case for both dismutase-active and -inactive mutants (22). Thus, only if SOD1 mutants increase Nox2 activity independently of their metalated state could mutant SOD1enhanced Rac1 activation and Nox-dependent $\mathrm{O}_{2}{ }^{-}$production be a feature common to the broader set of ALS-linked SOD1 mutants. This remains to be tested.

\section{The Nox inhibitor apocynin increases survival in ALS mice}

Decreasing ROS production by deleting Nox2 has already proven efficacious in ALS mice $(13,14)$. In the current study (20), administration of apocynin, an inhibitor of Nox activity, provided a therapeutic test of clinical relevance. The addition of apocynin to the drinking water of SOD $1^{\mathrm{G} 93 \mathrm{~A}}$ ALS mice beginning at 2 weeks of age increased the animals' lifespan in a dosedependent manner; at the highest dose, survival was increased by almost 4 months, 3 months of which was provided by slowing disease onset. The survival benefit was comparable to - and, in fact, greater than the deletion of the Nox2 gene in SOD1 $193 \mathrm{~A}$ mice previously reported by the same group (14). Apocynin is believed to block the translocation of $\mathrm{p} 47 \mathrm{phox} / \mathrm{p} 67 \mathrm{phox}$ to Nox (23) and would therefore inhibit only the Nox proteins requiring these cytosolic activators, which include Nox1 and Nox2. Of particular interest, apocynin only affected the production of $\mathrm{O}_{2}{ }^{-}-$by a glial cell line, but not a neuronal cell line, when cells were transfected with mutant SOD1 (20), providing support that apocynin effectiveness may be acting on glial cells - most likely microglia. Although expression of Nox 2 by neurons, astrocytes, and endothelial cells has been reported (15), staining for Nox2 in ALS mice only revealed accumulation in microglial cells (13). However, other Nox proteins are expressed in the CNS, including Nox1 in neurons and endothelial cells, Nox4 in astrocytes, neurons, and endothelial cells, and Nox5 in endothelial cells; moreover, high doses of apocynin have been reported to inhibit Nox4 and Nox5 (15). Therefore, apocynin could also act on cell types other than microglia.

Of relevance for patients, Nox2 upregulation has been reported in spinal cords of sporadic ALS cases (13), but it is still unknown whether patients with SOD1 mutations generate similar or greater increases in Nox2 activity or expression.
It is also not clear whether treatment with apocynin reduces overall microglial activation. Two reported studies of ALS mice with a Nox2 deletion reached divergent conclusions: one group described downregulation of microglial activation (14), and the other reported no difference in activation (13). It is not yet known how apocynin affects inflammation at comparable states in the progression of disease and whether apocynin affects mutant SOD1 expression.

As to the potential use of apocynin in human therapy, 2 primary questions remain. The obvious first question relates to safety. Mutations altering the activity of Nox2 (or other subunits of Nox) are known to cause chronic granulomatous disease (CGD) (15), which is characterized by severe and chronic infections that are hard to treat due to the incapacity of immune cells to produce $\mathrm{O}_{2}{ }^{-}-$and therefore to fight pathogens. CGD patients are usually chronically administered antibiotics and IFN- $\gamma$. In both studies from the Engelhardt group $(14,20)$, their ALS animals with lowered Nox activity developed eye infections that, if not treated with antibiotics, led to rapid death of the animals before paralysis. This infection was often seen before onset of disease and might therefore be a direct consequence of mutant SOD1 expression in the infected tissues deprived of Nox activity, rather than a consequence of impaired grooming behavior. A second issue regarding potential therapy with apocynin is the degree of disease slowing achieved when treatment is initiated after disease onset. In the current study (20), apocynin treatment of ALS mice at 80 days of age ( 45 days before typical end stage) slowed disease progression, but the effect was minor compared with treatment well before disease onset, which suggests that treatment may only be effective if started very early in the disease course. An additional explanation for this modest effect would be that treating all the animals at the same age does not take into account potential variations in disease onset for each animal. Initiation of treatment of individual mice at phenotypic onset is probably the more relevant guide for success in patient therapy, regardless of age, as previously described by Crow and colleagues (1). The finding that apocynin, whose primary target seems to be glial cells, slowed disease onset when administered early also implies that inflammation, at least in ALS mice, must happen earlier than commonly thought (before obvious microglial activation) and hints at a pri- mary involvement of microglial cells early after disease initiation.

With this new finding that interaction of metalated SOD1 with Rac1 serves to activate Nox 2 and that mutant forms of SOD1 amplify production of ROS by locking Nox 2 in its activated state, Harraz and colleagues have advanced a new hypothesis for the gain of toxic function of mutant SOD1 (20). Indeed, normal SOD1 function, typically defined only by its dismutase activity, is now implicated in other cellular pathways. Loss or gain of these additional properties could be induced by mutations in SOD1 as one of the toxic contributors in ALS.

Address correspondence to: Don W. Cleveland, Ludwig Institute for Cancer Research and Departments of Medicine and Neuroscience, CMM-E 3057, University of California at San Diego, 9500 Gilman Drive, La Jolla, California 92093, USA. Phone: (858) 534-7811; Fax: (858) 534-7659; E-mail: dcleveland@ucsd.edu.

1. Crow, J.P., Calingasan, N.Y., Chen, J., Hill, J.L., and Beal, M.F. 2005. Manganese porphyrin given at symptom onset markedly extends survival of ALS mice. Ann. Neurol. 58:258-265.

2. Boillee, S., Vande Velde, C., and Cleveland, D.W. 2006. ALS: a disease of motor neurons and their nonneuronal neighbors. Neuron. 52:39-59.

3. Clement, A.M., et al. 2003. Wild-type nonneuronal cells extend survival of SOD1 mutant motor neurons in ALS mice. Science. 302:113-117.

4. Rothstein, J.D., Jin, L., Dykes-Hoberg, M., and Kuncl, R.W. 1993. Chronic inhibition of glutamate uptake produces a model of slow neurotoxicity. Proc. Natl. Acad. Sci. U. S. A. 90:6591-6595.

5. Di Giorgio, F.P., Carrasco, M.A., Siao, M.C., Maniatis, T., and Eggan, K. 2007. Non-cell autonomous effect of glia on motor neurons in an embryonic stem cellbased ALS model. Nat. Neurosci. 10:608-614.

6. Nagai, M., et al. 2007. Astrocytes expressing ALSlinked mutated SOD1 release factors selectively toxic to motor neurons. Nat. Neurosci. 10:615-622.

7. Van Damme, P., et al. 2007. Astrocytes regulate GluR2 expression in motor neurons and their vulnerability to excitotoxicity. Proc. Natl. Acad. Sci.U.S. A. 104:14825-14830.

8. Kriz, J., Nguyen, M.D., and Julien, J.P. 2002. Minocycline slows disease progression in a mouse model of amyotrophic lateral sclerosis. Neurobiol. Dis. 10:268-278.

9. Van Den Bosch, L., Tilkin, P., Lemmens, G., and Robberecht, W. 2002. Minocycline delays disease onset and mortality in a transgenic model of ALS. Neuroreport. 13:1067-1070.

10. Nguyen, M.D., D’Aigle, T., Gowing, G., Julien, J.P., and Rivest, S. 2004. Exacerbation of motor neuron disease by chronic stimulation of innate immunity in a mouse model of amyotrophic lateral sclerosis. J. Neurosci. 24:1340-1349.

11. Boillee, S., et al. 2006. Onset and progression in inherited ALS determined by motor neurons and microglia. Science. 312:1389-1392.

12. Beers, D.R., et al. 2006. Wild-type microglia extend survival in PU.1 knockout mice with familial amyotrophic lateral sclerosis. Proc. Natl. Acad. Sci. U. S. A. 103:16021-16026.

13. Wu, D.C., Re, D.B., Nagai, M., Ischiropoulos, 


\section{commentaries}

H., and Przedborski, S. 2006. The inflammatory NADPH oxidase enzyme modulates motor neuron degeneration in amyotrophic lateral sclerosis mice. Proc. Natl. Acad. Sci. U. S. A. 103:12132-12137.

14. Marden, J.J., et al. 2007. Redox modifier genes in amyotrophic lateral sclerosis in mice. J. Clin. Invest. 117:2913-2919.

15. Bedard, K., and Krause, K.H. 2007. The NOX family of ROS-generating NADPH oxidases: physiology and pathophysiology. Physiol. Rev. 87:245-313.

16. Walder, C.E., et al. 1997. Ischemic stroke injury is reduced in mice lacking a functional NADPH oxidase. Stroke. 28:2252-2258.
17. Wu, D.C., et al. 2003. NADPH oxidase mediates oxidative stress in the 1-methyl-4-phenyl-1,2,3,6tetrahydropyridine model of Parkinson's disease. Proc. Natl. Acad. Sci. U. S. A. 100:6145-6150.

18. Gao, H.M., Liu, B., and Hong, J.S. 2003. Critical role for microglial NADPH oxidase in rotenoneinduced degeneration of dopaminergic neurons. J. Neurosci. 23:6181-6187.

19. Qin, B., et al. 2006. A key role for the microglial NADPH oxidase in APP-dependent killing of neurons. Neurobiol. Aging. 27:1577-1587.

20. Harraz, M.M., et al. 2008. SOD1 mutations disrupt redox-sensitive Rac regulation of NADPH oxidase in a familial ALS model. J. Clin. Invest. 118:659-670. 21. Sato, T., et al. 2005. Rapid disease progression correlates with instability of mutant SOD 1 in familial ALS. Neurology. 65:1954-1957.

22. Subramaniam, J.R., et al. 2002. Mutant SOD1 causes motor neuron disease independent of copper chaperone-mediated copper loading. Nat. Neurosci. 5:301-307.

23. Stolk, J., Hiltermann, T.J., Dijkman, J.H., and Verhoeven, A.J. 1994. Characteristics of the inhibition of NADPH oxidase activation in neutrophils by apocynin, a methoxy-substituted catechol. Am.J. Respir. Cell Mol. Biol. 11:95-102. 